\title{
The Kramers-Heisenberg Formula and the Gunn-Peterson Trough
}

\author{
Kiehunn BaCH ${ }^{1,3}$ And HeE-Won LeE ${ }^{2}$ \\ ${ }^{1}$ Department of Astronomy, Yonsei University, 50 Yonsei-ro, Seodaemun, Seoul, Korea; kbach@yonsei.ac.kr \\ ${ }^{2}$ Department of Astronomy and Space Science, Sejong University, Seoul, Korea; hwlee@sejong.ac.kr \\ ${ }^{3}$ Department of Science Education, Seoul National University of Education, 96 Seocho-Joongang, Seocho, Seoul, Korea
}

Received July 31, 2014; accepted September 29, 2014

\begin{abstract}
Recent cosmological observations indicate that the reionized universe may have started at around $z=6$, where a significant suppression around Ly $\alpha$ has been observed from the neutral intergalactic medium. The associated neutral hydrogen column density is expected to exceed $10^{21} \mathrm{~cm}^{-2}$, where it is very important to use the accurate scattering cross section known as the Kramers-Heisenberg formula that is obtained from the fully quantum mechanical time-dependent second order perturbation theory. We present the Kramers-Heisenberg formula and compare it with the formula introduced in a heuristic way by Peebles (1993) considering the hydrogen atom as a two-level atom, from which we find a deviation by a factor of two in the red wing region far from the line center. Adopting a representative set of cosmological parameters, we compute the Gunn-Peterson optical depths and absorption profiles. Our results are quantitatively compared with previous work by Madau \& Rees (2000), who adopted the Peebles approximation in their radiative transfer problems. We find deviations up to 5 per cent in the Gunn-Peterson transmission coefficient for an accelerated expanding universe in the red off-resonance wing part with the rest wavelength $\Delta \lambda \sim 10 \AA$.
\end{abstract}

Key words: cosmology: theory — intergalactic medium - quasars: absorption lines — radiative transfer - galaxies: high-redshift

\section{INTRODUCTION}

Quasar absorption systems have been excellent tools to investigate the intergalactic medium (IGM), from which it has been well known that the IGM in the nearby universe is highly ionized (e.g., Peebles 1993). Since the universe after the recombination era $z \sim 1100$ should be mostly neutral, there must be some epoch when the universe began to be re-ionized. Intensive studies have been performed on the emergence of the first objects that ended the dark age of the universe. Numerical calculations adopting the cold dark matter models predict a reionization epoch at $z \sim 6-12$ (e.g., Gnedin \& Ostriker 1997).

Around this epoch a broad absorption trough in the blue part of Ly $\alpha$ is expected and regarded as a strong indicator of the reionization of the universe, which was predicted by Gunn \& Peterson (1965) and independently also by Scheuer (1965). With the advent of the Hubble Space Telescope and 8 meter class telescopes there have been extensive searches for the GunnPeterson trough in the spectra of high redshift objects. Remarkable contributions have been made by the Sloan Digital Sky Survey, from which a number of high redshift quasars with $z$ ranging from 4 to 6 have been found. According to recent results based on Keck spectroscopy of these high redshift quasars (Becker et al. 2001; Fan et al. 2006a), the flux level drop around Ly $\alpha$ is much higher for a quasar with $z=6.28$ than those for other quasars with $z<6$, which indicates

CORRESPONDING AUTHOR: H.-W. Lee that the reionization epoch may be found at around $z \sim 6$. During the last decade, numerous studies of cosmic reionization have been discussed by using analytic models (Madau, Haardt \& Rees 1999; Miralda-Escudé, Haehnelt \& Rees 2000; Wyithe \& Loeb 2003), and direct numerical simulations (Gnedin 2000; Sokasian et al. 2002; Razoumov et al. 2002; Ciardi et al. 2003; Iliev et al. 2006; Ahn et al. 2012). Comprehensive reviews of the cosmic reionization are found in the literature (see Loeb \& Barkana 2001; Ciardi \& Ferrara 2005; Fan et al. 2006b; Morales \& Wyithe 2010; Bromm \& Yoshida 2011; Fan 2012).

The exact computation of the flux drop around Ly $\alpha$ requires an accurate atomic physical estimation of the scattering cross section. Recent theoretical works on the calculation of the Gunn-Peterson trough were provided by Miralda-Escudé (1998), who adopted the formula that was introduced in a heuristic way using the second order time-dependent perturbation theory by Peebles (1993). The formula is derived based on the assumption that the hydrogen atom is a two-level atom, in order to describe the behavior of the scattering cross section that is approximated by the Lorentzian near resonance and yields a $\omega^{4}$-dependence in the low energy limit. As Peebles noted explicitly, due to the two-level assumption, the formula provides an inaccurate proportionality constant in the low energy limit, even though it correctly gives the $\omega^{4}$-dependence.

The accurate cross section should be obtained from the second order time-dependent perturbation theory treating the hydrogen atom as an infinitely many-level 
atom including the continuum free states, which is known as the Kramers-Heisenberg formula. The discrepancy between the two formulae will be significant in the far off-resonance regions where the contribution from the $n p(n>2)$ states including the continuum states becomes considerable. Therefore, in order to obtain an accurate Gunn-Peterson profile it is essential to investigate the exact scattering optical depth of a medium with a high neutral hydrogen column density.

In this paper, we present a reliable atomic physics relation that governs the scattering around Ly $\alpha$ by introducing the Kramers-Heisenberg dispersion relation and a simple fitting formula around Ly $\alpha$. With the welldefined cross section schemes, we compute the GunnPeterson trough profiles adopting a representative set of cosmological parameters with our choice of the reionization epoch and make quantitative comparisons with previous works.

\section{The Kramers-Heisenberg Formula}

The interaction of photons and electrons is described by the second order time-dependent perturbation theory, of which the result is summarized as the famous Kramers-Heisenberg dispersion relation (Kramers \& Heisenberg 1925). As is well illustrated in a typical quantum mechanics text (see Heitler 1954; Bethe \& Salpeter 1957; Sakurai 1967; Berestetskii et al. 1971), it may be written as

$$
\begin{aligned}
\frac{d \sigma}{d \Omega}(\omega)= & \frac{r_{0}^{2}}{m_{e}^{2} \hbar^{2}} \mid \sum_{I}\left[\frac{\omega\left(\vec{p} \cdot \hat{\epsilon}^{\left(\alpha^{\prime}\right)}\right)_{A I}\left(\vec{p} \cdot \hat{\epsilon}^{(\alpha)}\right)_{I A}}{\omega_{I A}\left(\omega_{I A}-\omega-i \Gamma_{I} / 2\right)}\right. \\
& \left.-\frac{\omega\left(\vec{p} \cdot \hat{\epsilon}^{(\alpha)}\right)_{A I}\left(\vec{p} \cdot \hat{\epsilon}^{\left(\alpha^{\prime}\right)}\right)_{I A}}{\omega_{I A}\left(\omega_{I A}+\omega\right)}\right]\left.\right|^{2}
\end{aligned}
$$

where $\left(\hat{\epsilon}^{\alpha}, \hat{\epsilon}^{\alpha^{\prime}}\right)$ are the polarization state vectors associated with the incident photon and outgoing photon, respectively, $\Gamma_{I}$ is the radiation damping term associated with the intermediate state $I, \omega$ is the incident angular frequency, $\omega_{I A}$ is the angular frequency of the transition between I and the ground state $A$, and $r_{o}=e^{2} /\left(m_{e} c^{2}\right)=2.82 \times 10^{-13} \mathrm{~cm}$ is the classical electron radius (e.g., Sakurai 1967).

Here, the electron is in the ground state $A$ before scattering and de-excites to the same state. The summation (and integration) should be carried over all the intermediate states $I$ including the infinite number of bound states and the free or continuum states. For hydrogen, the dipole moment matrix elements can be explicitly written using the hypergeometric function (e.g., Bethe \& Salpeter 1957; Karzas \& Latter 1961; Berestetskii et al. 1971).

In the blue part of $\operatorname{Ly} \alpha$, it is possible that the scattering atom may de-excite to the excited $2 s$ state by re-emitting a photon with much lower frequency than the incident photon. This Raman scattering is negligible near Ly $\alpha$ due to small phase space available for an outgoing photon. However, this process becomes important as the incident photon energy increases. In the range where the current work is concerned, the Raman scattering process is safely neglected.

Despite the existence of explicit analytic expressions for each matrix element that is the Kramers-Heisenberg formula for hydrogen, it is still cumbersome to use the formula as it is. The Lorentzian function as an exact solution of the first order perturbation theory (Weisskopf \& Wigner 1930) provides an excellent approximation at the line center,

$$
\sigma(\omega)=\frac{3 \lambda_{\alpha}^{2}}{8 \pi} \frac{\Gamma_{2 p}^{2}}{\left(\omega-\omega_{\alpha}\right)^{2}+\Gamma_{2 p}^{2} / 4},
$$

where $\Gamma_{2 p}=6.25 \times 10^{8} \mathrm{~s}^{-1}$ is the radiation damping constant associated with the Ly $\alpha$ transition. In principle, it provides a good description of physical processes, such as the natural line broadening, transition life time, and decay rates of the excited state during the radiation-atom interaction. However, the linear perturbation theory cannot give a proper solution for the transition probability for off-center scattering.

For extremely dense Ly $\alpha$ clouds, the line center is easily saturated and the broad damping part becomes important. In order to describe the broad damping profiles of the Ly $\alpha$ clouds, Peebles (1993) introduced a heuristic formula based on the two-level approximation,

$$
\sigma(\omega)=\frac{3 \lambda_{\alpha}^{2}}{8 \pi} \frac{\Gamma_{2 p}^{2}\left(\omega / \omega_{\alpha}\right)^{4}}{\left(\omega-\omega_{\alpha}\right)^{2}+\Gamma_{2 p}^{2}\left(\omega / \omega_{\alpha}\right)^{6} / 4} .
$$

In his qualitative analysis, resonance scattering is smoothly connected to the off-center scattering with the $\omega^{4}$-dependency of classical Rayleigh scattering. As he pointed out, since the contribution of all possible intermediate states is neglected, the two-level approximation results in significant underestimation of the transition probabilities (by a factor of 2 in the low energy limit).

In previous studies, fitting relations for Rayleigh scattering have been introduced. Through their quantal analysis, Gavrila (1967) provided the fitting polynomial

$$
\frac{\sigma(\omega)}{\sigma_{T}}=0.4\left(\frac{\omega}{\omega_{\alpha}}\right)^{4}+0.9\left(\frac{\omega}{\omega_{\alpha}}\right)^{6}+12.6\left(\frac{\omega}{\omega_{\alpha}}\right)^{14}
$$

with the threshold frequency $(\lambda>1410 \AA)$, where $\sigma_{T}=8 \pi r_{0}^{2} / 3$ is the Thomson scattering cross section. In particular, Ferland (2001) used Gavrila's fit in his photoionization code CLOUDY. Earlier, Baschek \& Scholz (1982) has also introduced a similar expression in the wavelength range $\lambda>2000 \AA$. However, their fitting relations are not smoothly connected to resonance scattering (the Lorentzian), and exhibit about $4 \%$ occasional errors. Moreover, no simple polynomial fits to the blue wing of Ly $\alpha$ are available.

Instead, we investigate an analytic correction function which corrects the classical approximation in practical applications. Adopting a non-linear regression, a reliable correction function and its coefficients have been numerically determined. Between the wavelength interval $1100 \AA<\lambda<8000 \AA$ which covers the whole 

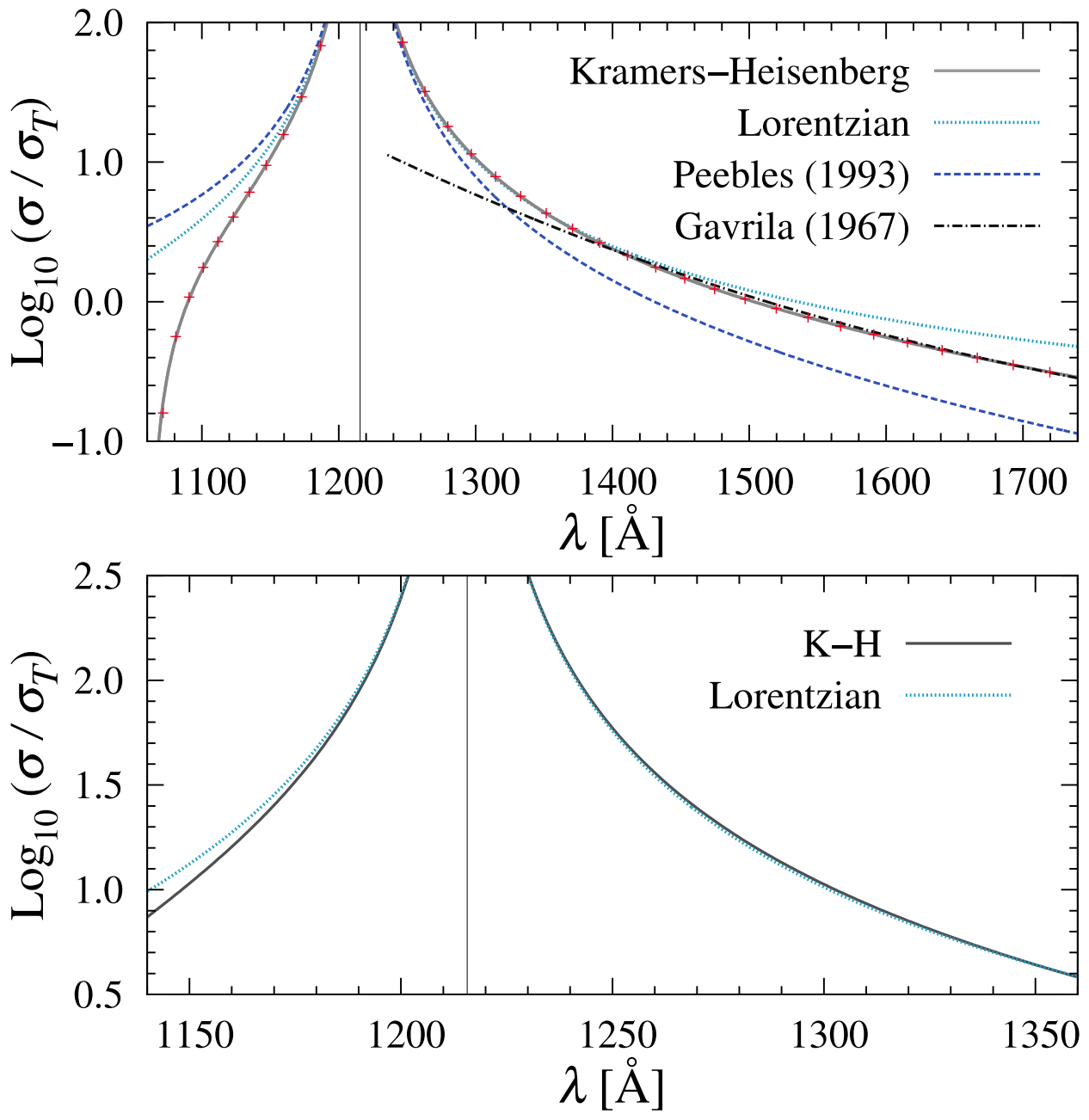

Figure 1. The scattering cross section around Ly $\alpha$. In the top panel, the intrinsic cross section known as the KramersHeisenberg formula (solid) is compared with the classical approximations, such as the Lorentzian (dotted) and the Peebles approximation (dashed). For the long wavelength scattering, Gavrila (1967) introduced a fitting function (dot-dashed) with a threshold wavelength $\lambda>1410 \AA$ and occasional errors (4\%). At the line center, the Lorentzian gives an excellent approximation for resonance scattering. Due to its slow damping rate, however, it seriously overestimates the scattering probabilities for low energy photons. In the case of the Peebles approximation, resonance scattering is smoothly connected to the classical Rayleigh scattering ( $\omega^{4}$-dependency). However, the two-level assumption leads to significant underestimation of the transition probability by neglecting all possible intermediate states. The crosses denote our analytic function which corrects the classical approximations to the Kramers-Heisenberg dispersion relation (Equations (5) and (6)). This fitting function is available in the wavelength range $1100 \AA<\lambda<8000 \AA$ which practically covers the Ly $\alpha$ domain with an excellent accuracy $(<1 \%$ errors). In the bottom panel, near-center scattering profiles are presented. It is notable that the Lorentzian underestimates scattering probabilities in wavelength region $\lambda_{0}<\lambda<1353 \AA$, which may cause an asymmetry problem in identifying line profiles of the DLAs.

practical wavelength domain of $\operatorname{Ly} \alpha$, our correction function has the form of

$$
\sigma(\omega)=\sigma_{L} \frac{4\left(\omega / \omega_{0}\right)^{4}}{\left(1+\omega / \omega_{0}\right)^{2}}[1+f(\omega)],
$$

where $\sigma_{L}$ is the Lorentzian function (Equation (2)), and $f(\omega)$ is deviation between the Kramers-Heisenberg formula and the cross section of the classical Rayleigh scattering. The deviation function has been fitted by the analytic form

$$
f(\omega)=a\left(1-e^{-b x}\right)+c x+d x^{2}
$$

with the coefficients

$$
(a, b, c, d)=(0.376,7.666,1.922,-1.036),
$$

where $x=1-\left(\omega / \omega_{0}\right)$. Our fitting relation satisfies two asymptotic properties: At the resonance frequency $\left(\omega / \omega_{0} \rightarrow 1\right)$, it approaches the Lorentzian function $(f(\omega) \rightarrow 0)$. For the long wavelength limit of the Ly $\alpha$ scattering, it properly corrects the classical Rayleigh scattering $(<0.5 \%$ error $)$. Moreover, it also extends to the blue wing of $\operatorname{Ly} \alpha$ with excellent accuracy $(<1 \%$ error).

Our formula for the full quantum mechanical cross section has been compared with the classical approx- 
imations and the fitting relations. In Figure 1, the Ly $\alpha$ cross section of off-center region (top) and nearresonance (bottom) is presented. The solid line denotes the Kramers-Heisenberg relation, the Lorentzian (dotted), the Peebles approximation (dashed), Gavrila's fit (dot-dashed), and our fitting function (crosses), respectively. The Lorentzian provides an excellent approximation at the resonance. However, its slow damping rate $\left(\sim \omega^{-2}\right)$ leads to overestimation of scattering probabilities at low energies. This asymptotic behavior implies that the radiation damping phenomenon can not be properly described by linear perturbation theory. In the bottom panel, it is notable that the deviation of the Lorentzian is slightly anti-symmetric with respect to the line center. The Lorentzian underestimates transition probabilities in the wavelength region $\lambda_{0}<\lambda<1353 \AA$. The largest deviation in this interval is about $3.5 \%$ underestimation at $\lambda=1274$ $\AA$, which may cause an asymmetry problem in identification of the DLAs line profiles (Lee 2003). Especially, $1 \%$ deviation of the Lorentzian from the intrinsic cross section is presented at a wavelength shift $\Delta \lambda=\lambda-\lambda_{\alpha}= \pm 3.3 \AA$ where the corresponding cross section is $\sigma=3.8 \times 10^{-21} \mathrm{~cm}^{2}$. This implies that the Voigt fitting has only $99 \%$ accuracy for a column density smaller than $3 \times 10^{20} \mathrm{~cm}^{-2}$.

In the case of the Peebles approximation (Equation (3)), resonance scattering is smoothly connected to the Rayleigh part. At low energies $\left(\omega \ll \omega_{\alpha}\right)$, it produces $\omega^{4}$-dependence which corresponds to the classical result. On the other hand, it also becomes the Lorentzian at the line center $\left(\omega \rightarrow \omega_{0}\right)$. However, the two-level assumption leads to underestimation of the transition probability by neglecting all possible intermediate states. Basically, oscillator strength is a good measure of contribution of individual excited state. For low energy photons $\left(\omega<\omega_{0}\right)$, the contribution of the $2 p$ state is comparable to that of the whole remaining states. Therefore, the Peebles approximation cannot provide an accurate description of the radiation damping phenomenon.

\section{Gunn-Peterson Absorption Profiles}

Combining the accurate $\operatorname{Ly} \alpha$ cross section with standard cosmology, we compute intergalactic absorption profiles of the reionization epoch. The presence of neutral hydrogen during reionization is signalled by the suppressed spectra around Ly $\alpha$, that is, the GunnPeterson troughs (Gunn \& Peterson 1965; Scheuer 1965). The ionizing radiation from the first luminous objects was scattered by the intervening neutral IGM. Then, even a small amount of neutral fraction $\left(\sim 10^{-4}\right)$ can produce a considerable Ly $\alpha$ opacity (Loeb \& Barkana 2001; Fan et al. 2006b).

We compute the Gunn-Peterson optical depth defined by

$$
\tau_{G P}=\int_{z_{r}}^{z_{s}} d z \frac{d l}{d z} \sigma\left[\nu=c(1+z) / \lambda_{o b s}\right] n(z),
$$

using the Kramers-Heisenberg formula and make comparisons with previous works performed by MiraldaEscudé (1998); Loeb \& Rybicki (1999); Madau \& Rees (2000). Here, $\lambda_{o b s}$ is the observed wavelength, $z_{r}, z_{s}$ are the redshifts of the complete reionization of the universe and the reionizing source, respectively, and $n(z)=n_{0}(1+z)^{3}$ is the homogeneous neutral hydrogen density at redshift $z$. We choose $z_{r}=6, z_{s}=7$ as in Madau \& Rees (2000), but do not consider the proximity effect of the ionizing source. The Gunn-Peterson optical depth can be written as

$$
\begin{aligned}
\tau_{G P}= & N_{\mathrm{HI}, 0} \int_{z_{r}}^{z_{s}} d z \sigma\left[\nu=c(1+z) / \lambda_{o b s}\right] \\
& \times(1+z)^{2} /\left[\Omega_{M}(1+z)^{3}+\Omega_{\Lambda}\right]^{1 / 2},
\end{aligned}
$$

where $\Omega_{M}, \Omega_{\Lambda}$ are the density parameters due to matter and the cosmological constant and the characteristic hydrogen column density defined as

$$
N_{\mathrm{HI}, 0} \equiv \frac{n_{0} c}{H_{0}} \text {. }
$$

Following previous work (Madau \& Rees 2000), we choose the present Hubble constant and the hydrogen number density $H_{0}=50 \mathrm{~km} \mathrm{~s}^{-1} \mathrm{Mpc}^{-1}, n_{0}=$ $2.4 \times 10^{-7} \mathrm{~cm}^{-3}$ so that $N_{\mathrm{HI}, 0}=4.3 \times 10^{21} \mathrm{~cm}^{-2}$.

In Figure 2, the Gunn-Peterson transmission coefficient defined as $T_{G P} \equiv \exp \left(-\tau_{G P}\right)$ is presented. We assumed two cosmological models: (top panel) the Einstein-de Sitter universe $\left(\Omega_{M}, \Omega_{\Lambda}\right)=(1,0)$ and (bottom panel) the $\Lambda$-cold dark matter universe $\left(\Omega_{M}, \Omega_{\Lambda}\right)=(0.35,0.65)$. In terms of the characteristic Gunn-Peterson optical depth $\tau_{G P, O}$ defined as

$$
\tau_{G P, 0} \equiv \frac{3 \lambda_{\alpha}^{3} \Gamma_{2 p} n\left(z_{s}\right)}{8 \pi H\left(z_{s}\right)},
$$

our choice of parameters in the case of the top panel yields the value of $\tau_{G P, O}=3 \times 10^{5}$ at $z_{s}=7$ as was adopted in the work of Madau \& Rees (2000). The horizontal axis represents the logarithm of the normalized wavelength ratio $\delta$ defined as

$$
\delta \equiv \frac{\lambda_{o b s}}{\lambda_{\alpha}\left(1+z_{s}\right)}-1
$$

thus $\delta=0$ corresponds to the resonance wavelength of Ly $\alpha$. The dotted lines represent the Gunn-Peterson transmission coefficient based on Equation (3). The deviation between the two formulae is notable around $\delta=10^{-2}$, where the deviation is about 3 per cent in the top panel and 5 per cent in the bottom panel. Peebles' approximation turns out to be pretty good for contemporary applications. However, the deviation between the two formulae will increase as $n_{0}$ or $N_{\mathrm{HI}, 0}$ increases, because the discrepancy of the Kramers-Heisenberg formula and the Peebles approximation becomes larger as the frequency is further away from the line center. Near resonance, both formulae are excellently approximated by the same Lorentzian. Therefore, no significant deviation is expected when the neutral medium is of low 


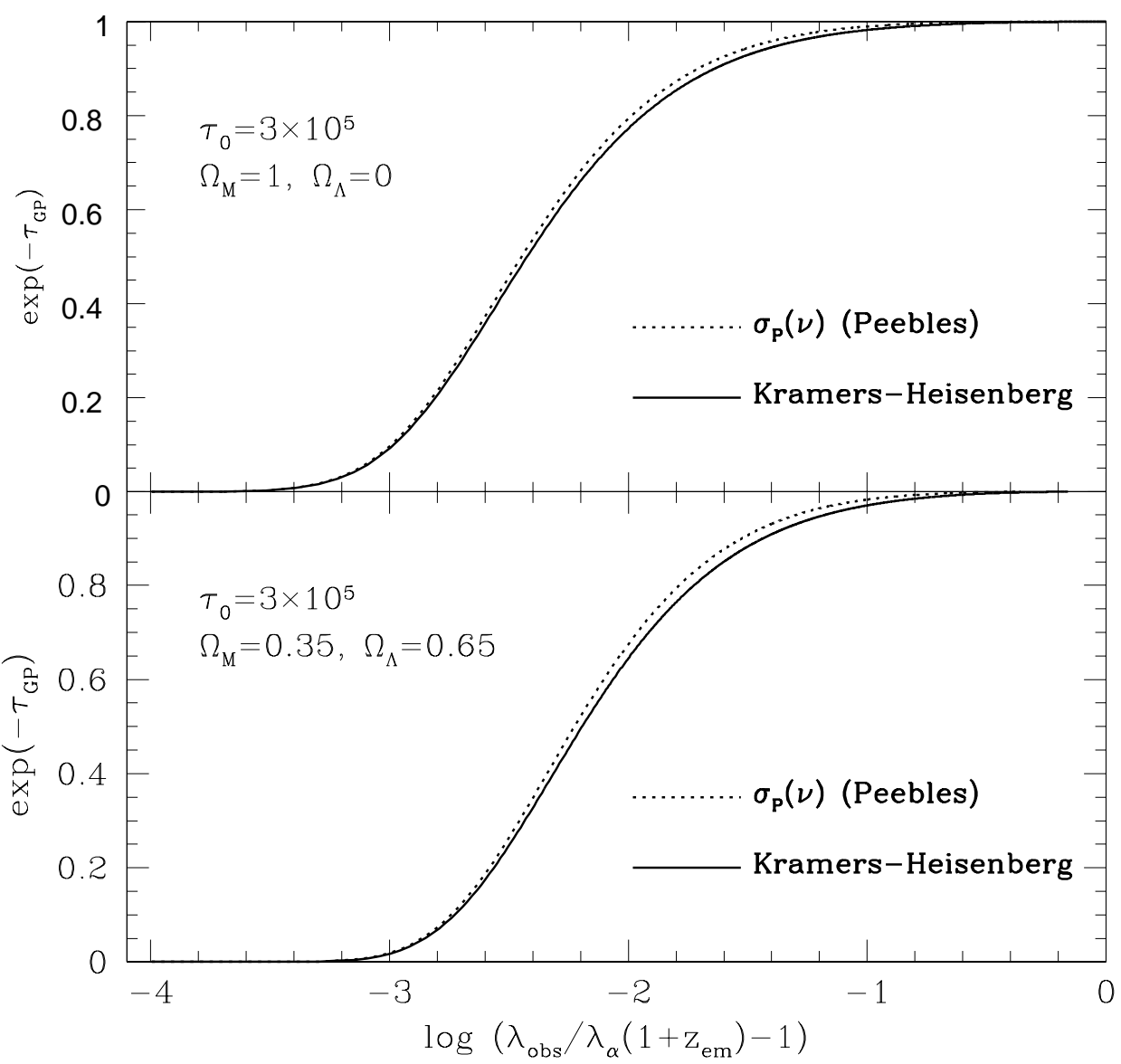

Figure 2. The Gunn-Peterson transmissions. Assuming two cosmological models: the flat matter-only universe with $\Omega_{M}=$ $1, \Omega_{\Lambda}=0$ (top panel) and the $\Lambda$-cold dark matter universe with $\Omega_{M}=0.35, \Omega_{\Lambda}=0.65$ (bottom panel), the Gunn-Peterson transmissions have been computed. The present Hubble constant and the hydrogen number density are chosen to be $H_{0}=50 \mathrm{~km} \mathrm{~s}^{-1} \mathrm{Mpc}^{-1}, n_{0}=2.4 \times 10^{-7} \mathrm{~cm}^{-3}$ so that $N_{\mathrm{HI}, 0}=n_{0} c H_{0}^{-1}=4.3 \times 10^{21} \mathrm{~cm}^{-2}$. The solid lines denote the transmitted flux using the Kramers-Heisenberg dispersion relation, and the dotted lines are for the values from the Peebles approximation (Equation (3)). In previous studies of the Gunn-Peterson model, the intergalactic absorption profiles have been calculated based on the Peebles approximation. Because of the two-level assumption, the Peebles approximation underestimates the scattering probabilities, which directly yields overestimation of the transmitted flux.

column density $\lesssim 10^{21} \mathrm{~cm}^{-2}$. It is notable that an accurate treatment of atomic physics is more important in an accelerated expanding universe where the universe was more compact than the universe without the cosmological constant.

In practice, the Gunn-Peterson test provides a unique tool which is highly sensitive to the line-of-sight distribution of neutral medium. Indeed, the Ly $\alpha$ absorption profiles can be affected by the line-of-sight distribution of neutral medium, or by a complex geometry of ionization fronts of $\mathrm{H}$ II bubbles. Adopting recent cosmological concordance values, we examine the effect of density distribution on the Gunn-Peterson profiles (Figure 3). Firstly, assuming a homogeneous distribution of the neutral IGM along the redshift interval $\left[z_{r}, z_{s}\right]=[6,8]$, the Ly $\alpha$ red wings are computed by using two cross section schemes of the Kramers-Heisenberg relation (solid) and the Peebles approximation (dotted). In addition, we also consider inhomogeneity of the lineof-sight distribution of the neutral medium by adjusting the fraction of neutral hydrogen $\left(f_{\mathrm{HI}}=n_{\mathrm{HI}} / n_{\mathrm{H}}\right)$. The (red) circle represents the transmitted flux based on the Kramers-Heisenberg formula but with the neutral fraction of $f_{\mathrm{HI}}=0.2$ in the redshift interval $[6.0,7.6]$ and with $f_{\mathrm{HI}}=1.0$ in $[7.6,8]$. This slab-shaped distribution where the neutral medium is highly localized is expected to be the last stage of cosmic reionization at $z=6 \sim 8$. In the blue part spectra of the rest frame of the source, transmitted fluxes are swept out by the high optical depth of the near-center scattering. Then, the neutral IGM at lower redshift than source location can affect the Ly $\alpha$ opacities in the far off-center of red damping wing with a small transition probability. This implies that a cumulative effect by the train of neutral hydrogen along the line-of-sight can be present in the far off-center region of the red damping wing. It also suggests that the classical approximation can not provide an accurate measurement of the cumulative effect or a proper continuum level of transmission. If the Ly $\alpha$ emitter is embedded in a mostly neutral IGM, the 


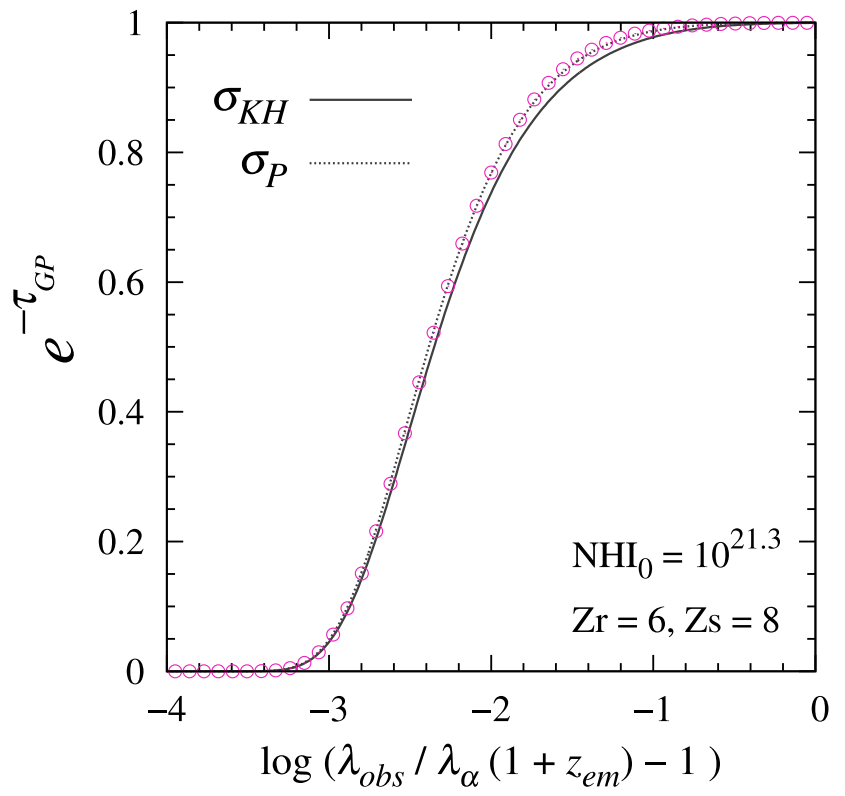

Figure 3. Comparison of the cross section schemes and the effect of the local distribution of neutral medium. Assuming concordance parameters $\left(\Omega_{\Lambda}, \Omega_{M}, h_{0}\right)=(0.75,0.25$, 0.71 ), the intergalactic absorption profiles have been calculated. Assuming a homogeneous distribution of the neutral IGM along the redshift interval $\left[z_{r}, z_{s}\right]=[6,8]$, we examine two cross section schemes: the Kramers-Heisenberg relation (solid) and the Peebles approximation (dotted). In addition, by adjusting the fraction of neutral hydrogen $\left(f_{\mathrm{HI}}=n_{\mathrm{HI}} / n_{\mathrm{H}}\right)$, a slab-shaped distribution where the neutral medium is highly localized is also considered. The (red) circle denotes transmitted flux based on the KramersHeisenberg relation with the distribution of $f_{\mathrm{HI}}=0.2$ in the redshift interval $[6.0,7.6]$ and $f_{\mathrm{HI}}=1.0$ in $[7.6,8]$.

Gunn-Peterson profile will give a substantial information of the density distribution near the source.

\section{SuMmary AND DISCUSSION}

In this paper, we have investigated the behavior of the scattering cross section around Ly $\alpha$ in a quantitative way, where the deviation from the Lorentzian becomes significant as the incident frequency gets further away from the line center. Therefore, in an analysis of the Gunn-Peterson trough profile, which is associated with a neutral medium with a high $\mathrm{HI}$, column density, an inaccurate treatment of the atomic physics of hydrogen may introduce significant errors in estimating important cosmological parameters including the epochs of the emergence of the first objects and the completion of the reionization of the universe.

Voigt profile fitting has been very successfully applied to quasar absorption systems with a broad range of $\mathrm{H}$ I column densities. However, the deviation of the true scattering cross section from the Lorentzian exceeds $1 \%$ when the relevant column density becomes $N_{\mathrm{HI}} \geq 3 \times 10^{20} \mathrm{~cm}^{-2}$ that is the typical column density of a damped Ly $\alpha$ absorber. This is especially important in some damped Ly $\alpha$ systems that may possess $N_{\mathrm{HI}}>10^{21} \mathrm{~cm}^{-2}$ (e.g., Turnshek \& Rao 1998).
Recently, numerous theoretical works of reionization processes have been studied by using analytic models (Madau, Haardt \& Rees 1999; Miralda-Escudé, Haehnelt \& Rees 2000; Wyithe \& Loeb 2003), and numerical simulations (Gnedin 2000; Sokasian et al. 2002; Razoumov et al. 2002; Ciardi et al. 2003; Iliev et al. 2006; Ahn et al. 2012). The intergalactic medium underwent a dramatic change in the chemical states and the morphological structures throughout the whole epoch of cosmic reionization (Loeb \& Barkana 2001; Fan et al. 2006b). Especially, the neutral column density distribuion from the DLAs is known to be highly inhomogeneous in the redshift range $1 \leq z \leq 5$ after reionization (Noterdaeme et al. 2012; Zafar et al. 2013). Moreover, recent spectroscopic observations of Lyman break galaxies (LBGs) in the redshift interval $6 \leq z \leq 7$ indicate a constant decrease of the Ly $\alpha$ emitter density (Ota et al. 2010; Pentericci et al. 2011). In their works, this decrease was interpreted as due to a combination effect by the early evolutionary stages of galaxies and by the local distribution of the remaining neutral medium.

\section{ACKNOWLeDgments}

We are grateful to the anonymous referee for a careful reading and constructive suggestions. This research was supported by the Basic Science Research Program through the National Research Foundation of Korea (NRF) funded by the Ministry of Education, Science and Technology (NRF-2010-0024990). KB gratefully acknowledges financial support of the Basic Science Research Program (NRF-2014-11-1019) and the BK Plus Research Program (21A-2013-15-00002).

\section{REFERENCES}

Ahn, K., Iliev, I. T., Shapiro, P. R., Mellema, G., Koda, J., \& Mao, Y. 2012, Detecting the Rise and Fall of the First Stars by Their Impact on Cosmic Reionization, ApJ, 756, L16

Baschek, B., \& Scholz, M. 1982, Astronomy and Astrophysics, Stars and Star Clusters Vol. 2b (Berlin: SpringerVerlag)

Becker, R. H., Fan, X., White, R. L., et al. 2001, Evidence for Reionization at $z \sim 6$ : Detection of a Gunn-Peterson Trough in a $z=6.28$ Quasar, AJ, 122, 2580

Berestetskii, V. B., Lifshitz, E. M., \& Pitaevskii, L. P. 1971, Relativistic Quantum Theory (Oxford: Pergamon Press)

Bethe, H. A., \& Salpeter, E. E. 1957, Quantum Mechanics of One and Two Electron Atoms (New York: Academic Press Inc.)

Bromm, V., \& Yoshida, N. 2011, The First Galaxies, ARA\&A, 49, 373

Ciardi, B., Stoehr, F., \& White, D. M. 2004, Simulating Intergalactic Medium Reionization, MNRAS, 343, 1101

Ciardi, B., \& Ferrara, A. 2005, The First Cosmic Structures and Their Effects, SSRv, 116, 625

Fan, X., Strauss, M. A., Richards, G. T., et al. 2006, A Survey of $z>5.7$ Quasars in the Sloan Digital Sky Survey. IV. Discovery of Seven Additional Quasars, AJ, 131, 1203

Fan, X., Carilli, C. L., \& Keating, B. 2006, Observational Constraints on Cosmic Reionization, ARA\&A, 44, 415 
Fan, X. 2012, Observations of the First Light and the Epoch of Reionization, RAA, 12, 865

Ferland, G. 2001, Hazy, A Brief Introduction to Cloudy 94.00

Gavrila, M. 1967, Elastic Scattering of Photons by a Hydrogen Atom, Phys. Rev., 163, 147

Gnedin, N. Y., \& Ostriker, J. P. 1997, Reionization of the Universe and the Early Production of Metals, ApJ, 486, 581

Gnedin, N. Y. 2000, Cosmological Reionization by Stellar Sources, ApJ, 535, 530

Gunn, J. E., \& Peterson, B. A. 1965, On the Density of Neutral Hydrogen in Intergalactic Space, ApJ, 142, 1633

Heitler, W. 1954, Quantum Theory of Radiation (Oxford: Oxford University Press)

Iliev, I. T., Mellema, G., Pen, U.-L., Merz, H., Shapiro, P. R., \& Alvarez, M. A. 2006, Simulating Cosmic Reionization at Large Scales - I. The Geometry of Reionization, MNRAS, 369, 1625

Karzas, W. J., \& Latter, R. 1961, Electron Radiative Transitions in a Coulomb Field, ApJS, 6, 167

Kramers, H. A., \& Heisenberg, W. 1925, Über die Streuung von Strahlung durch Atome, Z. Phys., 31, 681

Lee, H.-W. 2003, Asymmetric Deviation of the Scattering cross Section around Ly $\alpha$ by Atomic Hydrogen, ApJ, 594, 637

Loeb, A., \& Rybicki, G. B. 1999, Scattered Lyalpha Radiation around Sources before Cosmological Reionization, ApJ, 524, L527

Loeb, A., \& Barkana, R. 2001, The Reionization of the Universe by the First Stars and Quasars, ARA\&A, 39, 19

Madau, P., Haardt, F., \& Rees, M. J. 1999, Radiative Transfer in a Clumpy Universe. III. The Nature of Cosmological Ionizing Sources, ApJ, 514, 648

Madau, P., \& Rees, M. J. 2000, The Earliest Luminous Sources and the Damping Wing of the Gunn-Peterson Trough, ApJ, 542, L69

Miralda-Escudé, J. 1998, Reionization of the Intergalactic Medium and the Damping Wing of the Gunn-Peterson Trough, ApJ, 501, 15

Miralda-Escudé, J., Haehnelt, M., \& Rees, M. J. 2000,
Reionization of the Inhomogeneous Universe, ApJ, 530, 1 Morales, M. F., \& Wyithe, S. 2010, Reionization and Cosmology with 21-cm Fluctuations, ARA\&A, 48, 127

Noterdaeme, P., Petitjean, P., Carithers, W. C., et al. 2012, Column Density Distribution and Cosmological Mass Density of neutral gas: Sloan Digital Sky Survey-III Data Release 9, A\&A, 547, 1

Ota, K., Iye, M., Kashikawa, N., et al. 2010, Lyalpha Emitters at $z=7$ in the Subaru/XMM-Newton Deep Survey Field: Photometric Candidates and Luminosity Functions, ApJ, 722, 803

Peebles, P. J. E. 1993, Principles of Physical Cosmology (Princeton: Princeton University Press)

Pentericci, L., Fontana, A., Vanzella, E., Castellano, M., et al. 2011, Spectroscopic Confirmation of $z \sim 7$ Lyman Break Galaxies: Probing the Earliest Galaxies and the Epoch of Reionization, ApJ, 743, 132

Razoumov, A. O., Norman, M. L., Abel, T., \& Scott, D. 2002, Cosmological Hydrogen Reionization with ThreeDimensional Radiative Transfer, ApJ, 572, 695

Sakurai, J. J. 1967, Advanced Quantum Mechanics (Reading, Massachusetts: Addison-Wesley Publishing Company)

Scheuer, P. A. G. 1965, A Sensitive Test for the Presence of Atomic Hydrogen in Intergalactic Space, Nature, 207, 963

Sokasian, A., Abel, T., \& Hernquist, L. 2002, The Epoch of Helium Reionization, MNRAS, 332, 601

Turnshek, D. A., \& Rao, S. 1998, QSO Damped Lyalpha Absorption Systems at Low Redshift and the Giant Hydrogen Cloud Model for Damped Ly $\alpha$, ApJ, 500, L115

Wyithe, S., \& Loeb, A. 2003, Reionization of Hydrogen and Helium by Early Stars and Quasars, ApJ, 586, 693

Weisskopf, V. F. I., \& Wigner, E. 1930, Berechnung der natürlichen Linienbreite auf Grund der Diracschen Lichttheorie, Z. Phys., 63, 54

Zafar, T., Péroux, C., Popping, A., et al. 2013, The ESO UVES Advanced Data Products Quasar Sample. I. Dataset and New $N_{\mathrm{HI}}$ Measurements of Damped Absorbers, A\&A, 556, 141 